\title{
Cryptogenic organizing pneumonia masquerading as lung carcinoma: A case report and review of the literature
}

\author{
JI-PING HUO ${ }^{1}$, CUI LIU ${ }^{2}$, BEI-BEI JIN ${ }^{3}$, FENG-XIA DUAN ${ }^{4}$, \\ SHENG-HUI MEI ${ }^{1}$, XIN-GANG LI $^{1}$ and ZHI-GANG ZHAO ${ }^{1}$ \\ ${ }^{1}$ Department of Pharmacy, Beijing Tiantan Hospital Affiliated to Capital Medical University, Beijing 100050; \\ ${ }^{2}$ Department of Pathology, Union Hospital, Tongji Medical College, Huazhong University of Science \\ and Technology, Wuhan, Hubei 430022; Departments of ${ }^{3}$ Respiratory Medicine and ${ }^{4}$ Ultrasonography, \\ Beijing Tiantan Hospital Affiliated to Capital Medical University, Beijing 100050, P.R. China
}

Received June 7, 2016; Accepted April 21, 2017

DOI: $10.3892 /$ etm.2017.5393

\begin{abstract}
Cryptogenic organizing pneumonia (COP) is a rare pulmonary disorder of unknown etiology. COP with hemoptysis as the primary presenting symptom has rarely been reported. The present study reported a case of COP that resembled lung carcinoma with hemoptysis as the only clinical symptom. The patient recovered well following thoracoscope surgery. A literature review of 119 COP cases between 1995 and 2015 was presented. Cough, fever and dyspnea were the most common clinical manifestations. The most common imaging manifestations were multiple or single consolidation, lung nodules, migratory sign, reversed halo sign, and multiple ground-glass opacity. A total of 3 cases exhibited COP accompanied by lung cancer. Glucocorticoids were effective for the majority of cases and invasive surgeries were implemented in most cases. The majority of cases recovered or relieved, and the prognosis of COP was relatively good. COP was easily confused with lung tumor and it is necessary to make differential diagnosis between COP and lung cancer. Invasive surgery should be avoided when possible to avoid or reduce patient trauma.
\end{abstract}

\section{Introduction}

Organizing pneumonia (OP), which was previously called bronchiolitis obliterans organising pneumonia, is a well-known clinicopathological entity (1-3) that is associated with non-specific clinical manifestations. Lange initially described OP in 1901 (2) and Epler et al (4) published the largest case series of OP in 1985. OP is considered a rare disease with an

Correspondence to: Dr Zhi-Gang Zhao, Department of Pharmacy, Beijing Tiantan Hospital Affiliated to Capital Medical University, 6 Tiantan Xili, Dongcheng, Beijing 100050, P.R. China

E-mail:1022zzg@sina.com

Key words: cryptogenic organizing pneumonia, lung carcinoma, hemoptysis incidence of 1.96/100,000 (3); however, incidence has reportedly been increasing since the 1980s (2).

OP may be characterized as primary or secondary OP based on etiology. Primary OP is referred to as cryptogenic organizing pneumonia (COP) (1), has no known cause and is classified as an idiopathic interstitial pneumonia. Secondary OP is associated with various factors, including pathological infection (especially by Mycoplasma pneumoniae, Chlamydia pneumoniae and various types of virus (5), connective tissue diseases, malignancies, administration of various agents, chemotherapy, radiotherapy, organ transplantation (3), the inhalation of harmful gases $(1,2)$ and certain occupations or environments (6). As OP may be idiopathic or associated with a known underlying disease, delayed diagnosis or mis diagnosis is likely to occur (7).

The characteristic clinical features of OP are typically non-specific and include constitutional symptoms with flu-like illness. Common clinical manifestations of OP include cough, dyspnea, fever and weight loss $(2,7)$. Hemoptysis has rarely been reported as the primary presenting symptom of OP (2). The present case report details a Chinese case of COP with hemoptysis as the primary and unique clinical manifestation.

A literature review was carried out and a total of 23 studies published between 1995 and 2015 were included. A total of 119 COP cases were compared and analyzed for clinical manifestations, imaging manifestations, treatment and prognosis. The results of the review indicated that diagnosis of COP with lung cancer was challenging and COP was sometimes treated excessively, for example surgery was performed when steroids would have been sufficient, however the majority of patients with COP had a relatively good prognosis.

\section{Case report}

A 61-year-old Chinese male patient was presented with bloody sputum at Beijing Tiantan Hospital, Beijing, China, in August 2015. The chest X-ray revealed an abnormal shadow. The patient had no other chronic diseases such as hypertension or diabetes and no history of alcohol abuse or smoking. The results of human immunodeficiency virus and hepatitis $\mathrm{B}$ virus testing were negative. 
Laboratory tests detected a white blood cell count of $4.92 \times 10^{9} / 1$ (normal range, $4.0-10.0 \times 10^{9} / 1$ ), neutrophil percentage of $58.8 \%$ (normal range, 51.0-75.0\%), lymphocyte percentage of $31.7 \%$ (normal range, 20-40\%), monocyte percentage of $8.1 \%$ (normal range, $3.0-8.0 \%$ ), eosinophil percentage of $1.0 \%$ (normal range, $0.5-5.0 \%$ ), basophils percentage of $0.4 \%$ (normal range, $0-1.0 \%$ ), red blood cell count of $4.88 \times 10^{12} / 1$ (normal range, 4.0-5.5x10 $12 / 1$ ), hemoglobin concentration of $145 \mathrm{~g} / \mathrm{l}$ (normal range, 120-160 g/l), platelet count of $184 \times 10^{9} / 1$ (normal range, 100-300) x10 $/ 1$ ) and C-reactive protein level of $0.04 \mathrm{mg} / \mathrm{dl}$ (normal range, 0.0-0.6 mg/dl). Hepatic and renal functions and glucose and lipid levels in blood circulation were within normal ranges. Carbohydrate antigen 125, cytokeratin-19 fragment, neuron-specific enolase, squamous cell carcinoma associated antigen, carcino embryonic antigen and pro-gastrin-releasing peptide levels were within normal ranges.

Chest enhanced computed tomography scanning revealed a $3.0 \times 1.1 \times 2.0 \mathrm{~cm}$ irregular mass with rough edges and inhomogenous density in the apical region of the right upper lobe (Fig. 1). Pleural pull signs were visible around the mass. The lung demonstrated limitations of pulmonary emphysema. No marked swelling was observed in the lymph nodes in the mediastinal area and bilateral hilum of the lung. There was no effusion in the bilateral thorax and pericardium. Positron emission tomography-computed tomography scan with fluorin-18 fluorodeoxyglucose further revealed a high radioactivity uptake of this agent at the mass and a maximum standardized uptake value of 14.1 was recorded (Fig. 1). The mass appeared lobulated with surrounding burrs, and pleural pull signs were observed in the surrounding area. Primary malignant tumor was suspected from the imaging results.

Bronchoscopy revealed a normal tracheal mucosa and lumen. Pulmonary function tests presented normal volume and ventilatory function. Electrocardiogram examination returned normal results.

A surgical procedure was performed for the diagnosis and treatment of the mass. A partial resection including the mass was performed prior to frozen section examination. The lesion tissue and surrounding normal tissue were removed and kept dry. Tissue blocks were placed on a cold stage for embedding with Richard-Allan Neg 50 frozen section embedding medium (Thermo Fisher Scientific Inc., Waltham, MA, USA). Following quick-freezing $\left(-25-30^{\circ} \mathrm{C}\right)$ for $2 \mathrm{~min}$ and samples were sliced into sections $3-5 \mu \mathrm{m}$ thick. Sections were then attached to slides and fixed with fixing fluid composed of $95 \mathrm{ml} \mathrm{95 \%} \mathrm{ethanol} \mathrm{and} 5 \mathrm{ml}$ glacial acetic acid (Sinopharm Chemical Reagent Co., Ltd., Shanghai, China) for 1-2 min at room temperature. Hematoxylin-eosin (Baso diagnostics Inc., Zhuhai, China) staining was then performed at room temperature for 8-12 min. Examination of frozen sections revealed infiltration by numerous inflammatory cells with fibrous tissue hyperplasia; however, no apparent malignant tumor was identified. Wedge resection of the upper lobe of the right lung was successfully implemented via thoracoscope surgery. On the day following surgery, bedside chest X-ray examination was performed, which confirmed that the mass was completely removed. Electrocardiogram monitoring and blood routine examination results were within normal ranges. Atomization inhalation treatment with ipratropium bromide solution (500 $\mu \mathrm{g}$; Shanghai Boehringer Ingerhan Pharmaceutical Co., Ltd, Shanghai, China) was administered 3 times per day for three days following surgery, Cefminox sodium (2 g, Qilu Pharmaceutical Co., Ltd, Jinan, China) was infused intravenously twice 1 day following surgery to prevent postoperative infection and Flurbiprofen axetil (100 mg, Beijing Ted Pharmaceutical Co., Ltd, Beijing, China) was infused intravenously twice per day two days following surgery for postoperative analgesia.

Pathological examinations revealed typical characteristics of OP. The alveoli and alveolar ducts were filled with plugs of granulation tissue, which were composed of fibroblasts. Furthermore, chronic inflammatory cell infiltration and a few scattered mononuclear macrophages were detected in tissue sections (Fig. 2).

The symptom of hemoptysis disappeared following the surgical resection and the patient recovered well. As no other lesions were detected in the lung, corticosteroid administration was not considered and follow-up observation was recommended for the patient. Informed consent was obtained from the patient prior to inclusion in the present report.

\section{Literature review}

Literature search. A search of the literature was performed using Medline (http://medline.com) with the keywords 'bronchiolitis obliterans organizing pneumonia OR cryptogenic organizing pneumonia AND lung cancer' and 'bronchiolitis obliterans organizing pneumonia OR cryptogenic organizing pneumonia AND case report'. Studies on secondary OP were excluded and a total of 23 studies $(1,2,5,6,8-26)$ were retrieved by December 2015 .

From these 23 studies, data was reviewed from 119 patients with COP diagnosed between 1995 and 2015 published in PubMed (http://www.ncbi.nlm.nih.gov/pubmed) and ELSEVIER (https://www.elsevier.com/). The 119 patients included 78 males and 41 females who ranged in age from 37-88 years. The characteristics of these cases are detailed in Table I. Pulmonary function tests typically revealed a mild to moderate restrictive pattern.

Clinical manifestations. Among the 119 reviewed cases, patient clinical symptom information was obtained from 114. Of these 114 cases, 20 (17.5\%) demonstrated no symptoms. Flu-like respiratory symptoms, such as cough and fever were the most common symptoms. Cases including cough accounted for $52.6 \%(n=60)$, fever accounted for $44.7 \%(n=51)$ and dyspnea accounted for $43.0 \%(n=49)$. Clinical manifestations were various and non-specific, including hemoptysis $(n=15 ; 13.2 \%)$, malaise $(n=10 ; 8.7 \%)$, wheezing $(n=9 ; 7.9 \%)$, chest pain $(n=4$; $3.5 \%)$, weight loss $(n=4 ; 3.5 \%)$, expectoration $(n=3 ; 2.6 \%)$, sweats $(n=2 ; 1.8 \%)$, fatigue $(n=2 ; 1.8 \%)$, anorexia $(n=1 ; 0.9 \%)$, depression $(n=1 ; 0.9 \%)$ and generalized muscle and joint pain $(\mathrm{n}=1 ; 0.9 \%)$. Although cases including hemoptysis accounted for $13.2 \%$, it rarely occurred alone and was typically accompanied by other symptoms. Joint pain and myalgia $(n=1,0.9 \%)$ were also infrequent (23).

Imaging manifestations. Imaging appearances of COP cases were polymorphous and non-specific, but various 


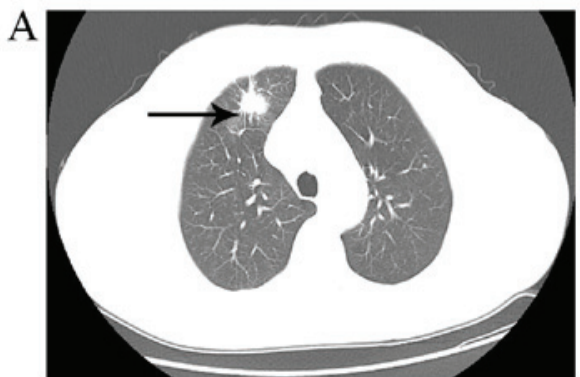

B

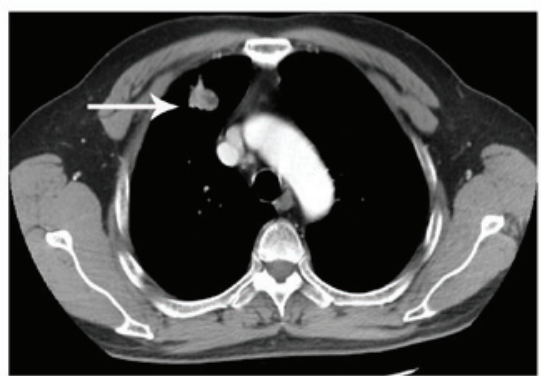

$\mathrm{C}$

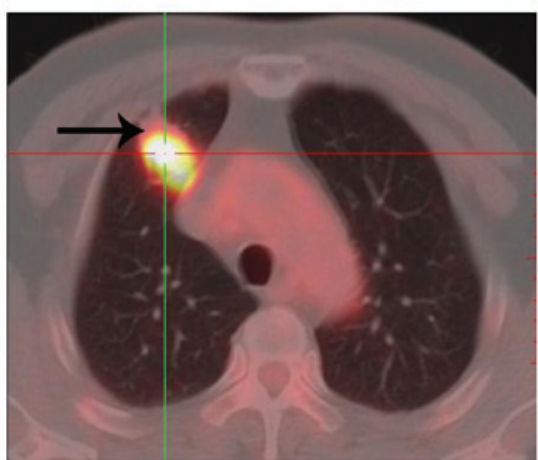

Figure 1. Radiological presentation. (A) Chest computed tomography viewed on the lung window (axial and coronal) indicated a $3.0 \times 1.1 \times 2.0 \mathrm{~cm}$ irregular mass (arrow) with rough edges, which is called 'spiculation sign' in the upper lobe of the right lung. Pleural pull signs were visible around the mass. (B) Mediastinal window of chest enhanced computed tomography scan indicated the inhomogenous density of the mass (arrow). (C) Positron emission tomography-computer tomography scanning of the chest indicated a strong accumulation of fluorin-18 fluorodeoxyglucose (arrow) in the upper lobe of the right lung and the maximum standardized uptake value (SUV) was 14.1.

presentation forms had been recently recognized as more characteristic to the diagnosis (26). In the 119 reviewed cases, the most common manifestations were multiple or single consolidation $(n=48 ; 40.3 \%)$, lung nodules $(n=43 ; 36.1 \%)$, migratory sign $(\mathrm{n}=34 ; 28.6 \%)$, reversed halo sign $(\mathrm{n}=33$; $27.7 \%)$, multiple ground-glass opacity $(\mathrm{n}=27 ; 22.7 \%)$, pleural effusion $(\mathrm{n}=18 ; 15.1 \%)$, multiple patchy opacities $(\mathrm{n}=8 ; 6.7 \%)$ and air bronchogram $(\mathrm{n}=5 ; 4.2 \%)$.

COP accompanied by lung cancer. Among these cases, 3 male patients were reported to have exhibited COP accompanied by lung cancer: A 73-year-old patient exhibited COP and adenocarcinoma (18), a 65-year-old patient exhibited COP and endobronchial squamous cell carcinoma (5), and a 60-year-old patient exhibited COP and bronchogenic carcinoma (13). These cases exhibited predominant clinical and radiological signs of COP $(5,13,18)$. Frequently, small foci of OP accompanied lung cancer infiltrations. OP was sometimes observed as a reaction to radiotherapy or chemotherapy, but it was rarely a predominant lesion in the course of lung cancer (5).
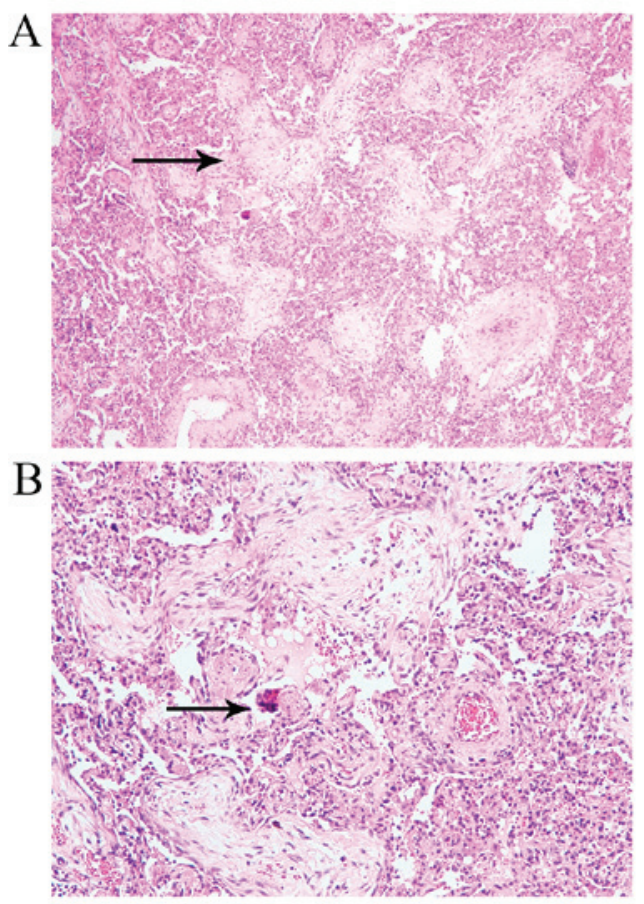

Figure 2. Pathological presentation presented using Hematoxylin and eosin staining. (A) Alveoli and alveolar ducts were filled with granulation tissue (arrow). Magnification, x40. (B) Alveolar spaces were occupied by fibrous tissue cells and multinuclear giant cells (arrow). Magnification, x100.

Due to the presentation of the two diseases being very similar, occasional misdiagnosis or missed diagnosis is inevitable. Morichika (27) et al reported a case of a 68-year-old man diagnosed with invasive mucinous adenocarcinoma of the lung. Computed tomography demonstrated subpleural ground-glass opacity and small nodules with cavitation. Repeated bronchoscopy revealed no atypical cells, but the surgical biopsy specimen was diagnostic for adenocarcinoma. Lung cancer should be excluded in the differential diagnosis of patients with clinical features of OP.

Diagnosis and treatment. Glucocorticoids were confirmed to be administered in $41 / 119$ (34.5\%) cases, and the majority of these cases exhibited a good clinical response, whereas only $2 / 41(4.9 \%)$ did not respond. Invasive surgeries were implemented in the majority of cases $(n=117 ; 98.3 \%)$ to make a definite diagnosis or for further treatment while information was not available on one of the remaining cases and the other was successfully treated using drugs alone. The number of patients diagnosed by cytology or pathology were as follows: Transbronchial lung biopsy, 4 (3.4\%); percutaneous lung biopsy, 2 (1.7\%); thoracoscopy, 4 (3.4\%); open lung biopsy, 6 (5.0\%); bronchoscopy and thoracotomy, 2 (1.7.\%); partial resection using an endoscopic linear cutter, $1(0.9 \%)$; percutaneous lung biopsy and open-chest partial excision, $1(0.9 \%)$; traditional surgical methods, including wedge resection and lobectomy, 23 (19.3\%); the full details of 3 (2.52\%) cases were not described; 1 case $(0.9 \%)$ was cured with drugs without biopsy; and 72 (60.5\%) cases in reference 20 were not clearly described. Considering the benign nature of OP and the efficacy of steroids, major pulmonary resections should be avoided (6). 


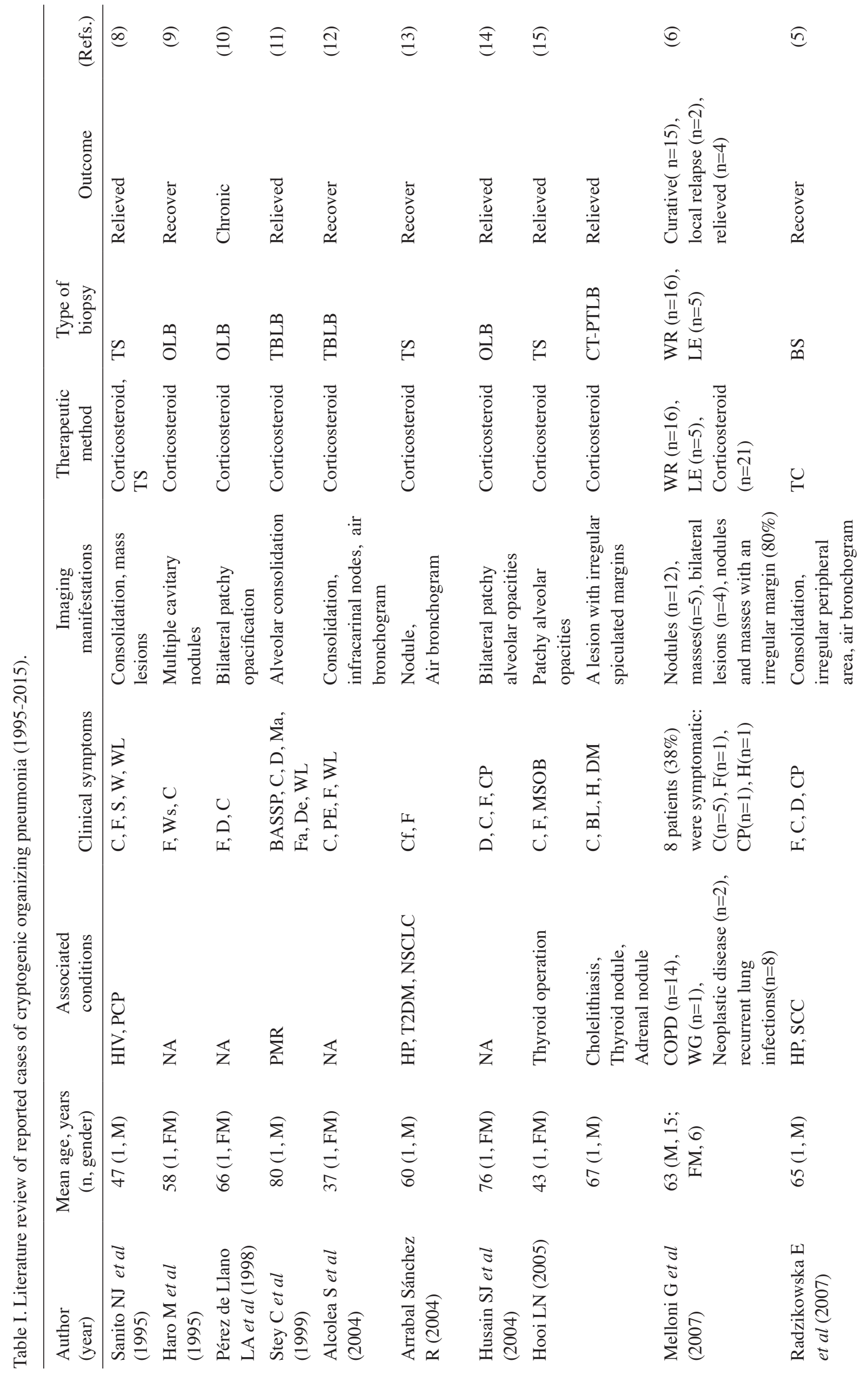




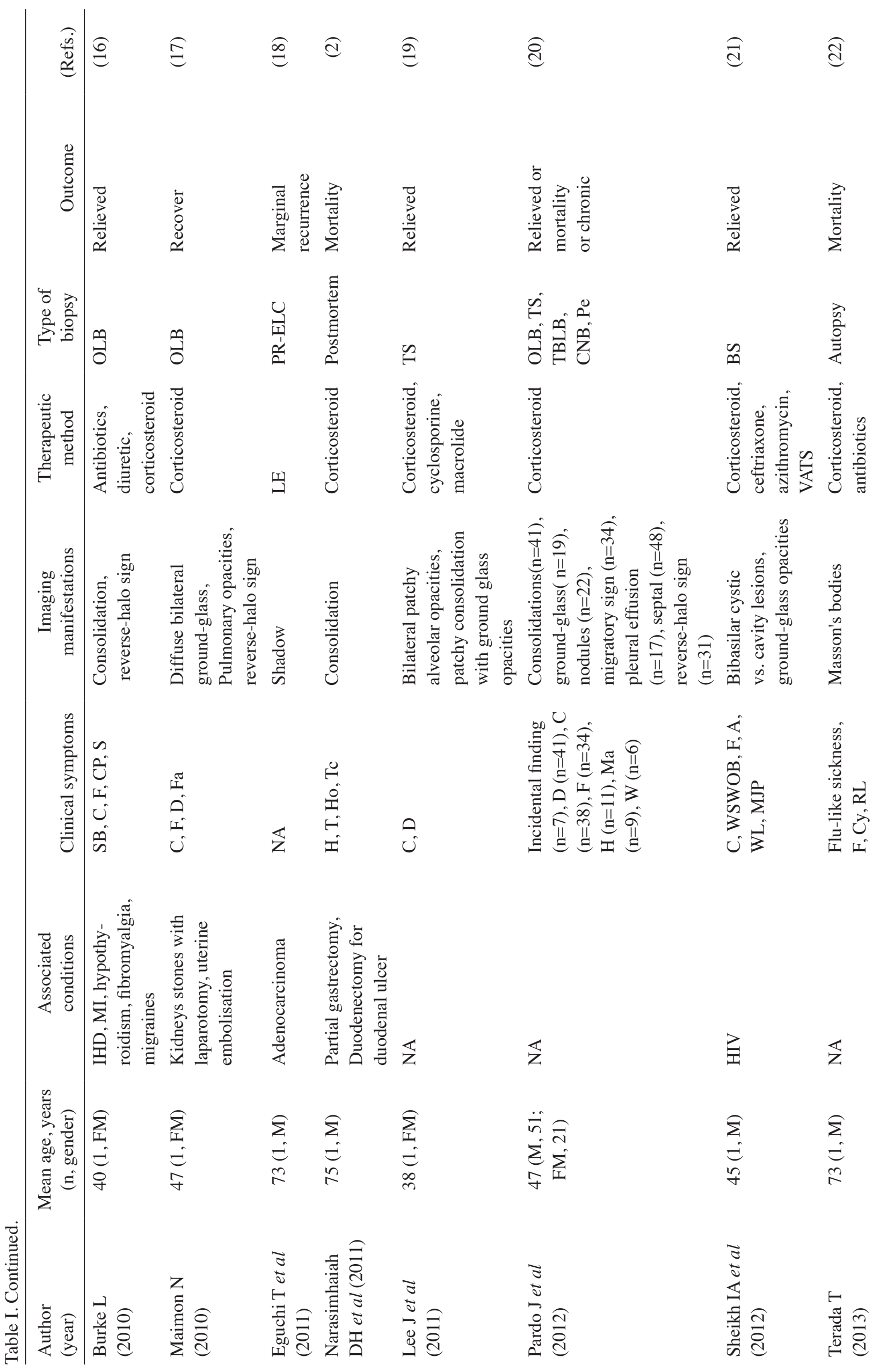




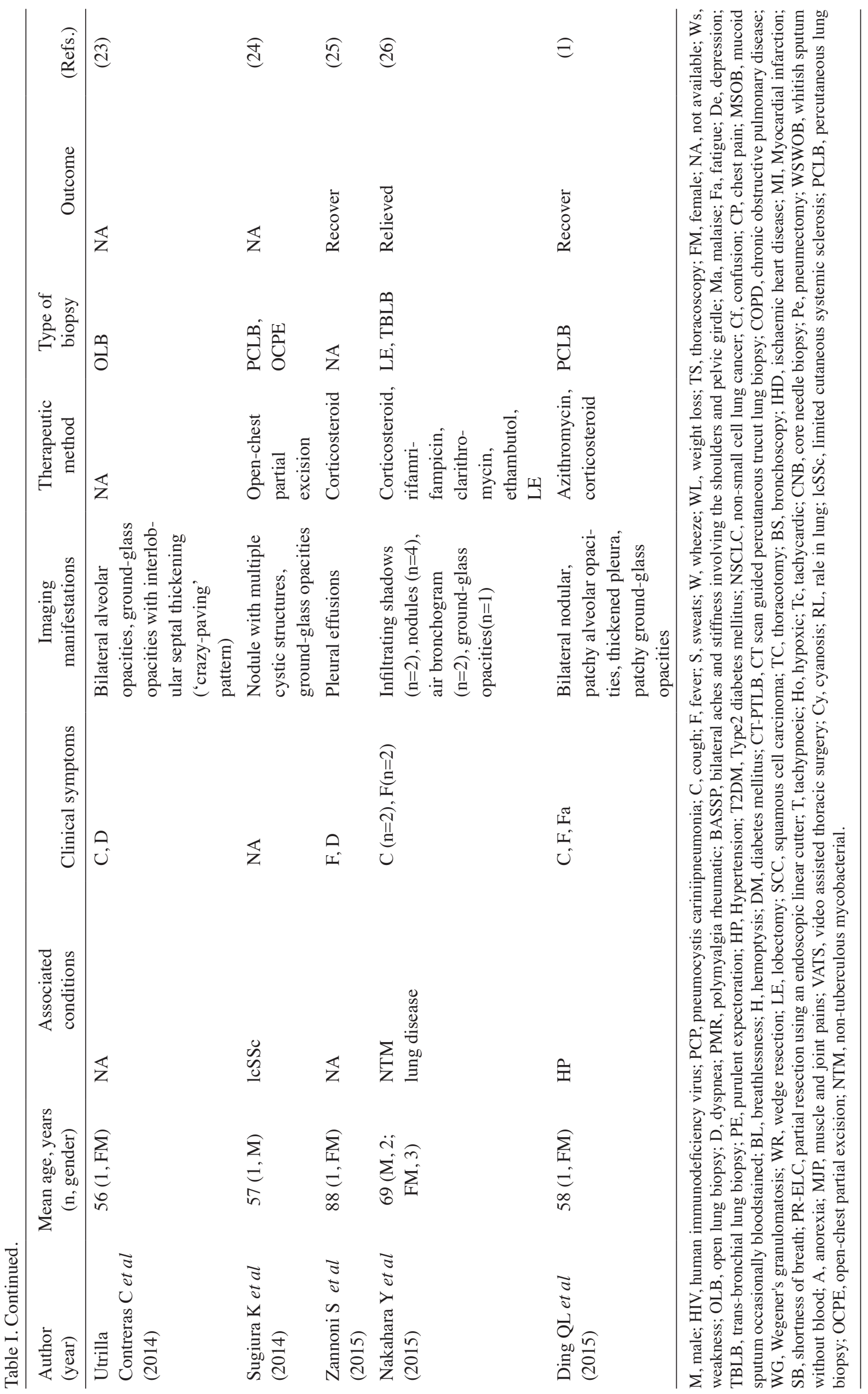


Among the 119 cases, 2 patients succumbed (1.7\%), the prognosis of 2 cases was not available (1.7\%), local recurrence occurred in 2 cases $(1.7 \%)$, marginal recurrence occurred in 1 case $(0.9 \%), 72$ cases $(60.5 \%)$ in reference 20 were not clearly described, but the majority of them recovered or were relieved. The remaining cases were recovered or relieved. Overall, the prognosis of COP was relatively good.

\section{Discussion}

COP is a rare disease that occurs in both men and women with reports increasing in frequency (26). The illness is not known to be associated with smoking (2). The diagnosis of OP requires a multidisciplinary approach combining clinical and radiological expertise, with histopathological evidence when a lung biopsy has been performed (26). Sometimes, OP may simulate lung cancer (18) or coexist with lung cancer (26). As such, it may be difficult but necessary to make differential diagnosis between OP and lung carcinoma.

OP and lung cancer typically exhibit similar clinical manifestations. Clinical manifestations of OP are non-specific. The typical presentation is of a subacute or chronic clinical course with fever, cough, weight loss and dyspnea, and less commonly with chest pain and hemoptysis (2), whereas lung cancer is typically characterized by chest pain and hemoptysis. For central-type lung cancer, clinical manifestations typically include irritating cough, dyspnoea, asthma, recurrent pneumonia, hemoptysis, and nerve compression symptoms (28). For peripheral lung cancer, chest pain, dyspnoea and pleural effusion are common clinical symptoms (28).

It is difficult to distinguish lung cancer and OP from imaging, as they are diseases characterized by abnormal pulmonary shadows $(5,6)$. Lung cancer typically shows irregular nodules or masses with an obscure boundary (3). Lobulation, spiculation, pleural invasion and lymph node metastases are also common clinical manifestations (28). OP may also present in the form of solid, mixed density, or, more rarely, ground-glass nodules. Typical radiological evidence of OP includes the presence of opacity, or consolidations with ground-glass appearance and lesions may migrate, with bilateral and peripheral distribution (29). Ground-glass opacity surrounding the OP lesion is a common sign, which typically indicates interstitial fibrosis and chronic inflammatory cell infiltration with a patchy area of polypoid granulation tissue (3). The most specific indicators of OP were peri lobular abnormalities, reverse halo sign, radial bands of consolidation containing an air bronchogram, and evolution and migration of the lesions over time $(5,26)$, whereas tumor lesions are stationary. Furthermore, lymph node enlargement and pleural fluid are rarely observed in OP, but are often reported in lung carcinoma.

The pathologic OP pattern typically occurs in patients with lung cancer (18). OP is characterized by granulation tissue plugs within the lumens of small airways extending into the alveolar ducts and airways $(2,3,7)$. Inflammatory debris fill the alveoli and spread to the alveolar ducts and terminal bronchioles, with characteristic endoluminal buds of granulation tissue known as Masson bodies (26). The clusters of granulation tissue, which are composed of fibroblasts and myofibroblasts, form as a result of non-specific tissue repair (23).

OP may accompany lung cancer as a result of the direct influence of a tumor on the surrounding lung parenchyma, or as a consequence of bronchial obstruction (5). Furthermore, anticancer treatment, including chemotherapy and radiotherapy, has been demonstrated to induce OP (5).

At present, the more common treatment methods are systemic corticosteroids therapy and surgical therapy. In 2013, an official statement from the American Thoracic Society/European Respiratory Society (30) recommended the following monitoring strategy of COP: Short-term observation to confirm treatment response and long-term observation to ensure that the response is preserved. Therefore, long-term follow-up observation is often necessary.

COP is not responsive to antibiotic therapy (7). Treatment with systemic corticosteroids typically achieves rapid improvement in symptoms and has a $>65 \%$ cure rate, without significant sequelae $(2,23)$. The most effective therapy is long-term administration of corticosteroids at high doses that are gradually increased during a treatment period of 6-12 months (29). However, when the corticosteroids are withdrawn or the dosage is decreased, COP may reoccur (7). In addition, prolonged treatment with corticosteroids is often associated with complications, including osteoporosis and trigger infection in diverse organs and systems, for example inhaling glucocorticoids may cause oral candidiasis infection, so patients are advised to use mouthwash after using inhaled glucocorticoids in order to avoid the occurrence of adverse reactions (7). Conversely, some patients may improve without treatment and spontaneous regressions have been observed $(3,5)$. Treatment regimens with corticosteroids are not completely defined at present (23).

Lung biopsy or a pulmonary resection is often required to enable a definitive diagnosis (26). Transbronchial lung biopsy and percutaneous lung biopsy are frequently used techniques, but sampling limitation from the two techniques may lead to misdiagnosis as small biopsy tissues may not represent the intrinsic lesion (25). A full resection of the lesions and histopathological examination of the larger organization are typically necessary (31). Furthermore, thoracoscopy has become a common surgical method (32). In view of OP's benign nature and the efficacy of steroid therapy, unnecessary pulmonary resection should be avoided (3).

In conclusion, the present report detailed a case of COP with hemoptysis as the primary clinical manifestation, which resembled and was mistaken for lung cancer. The patient recovered well following wedge resection of the upper lobe of the right lung via thoracoscope. The present case demonstrated that it is difficult to make differential diagnosis between OP and pulmonary carcinoma, and pulmonary carcinoma should be excluded prior to the diagnosis of OP.

$\mathrm{OP}$ is a non-specific inflammatory process caused by various injuries of either definite or idiopathic etiology. Its clinical symptoms and imaging manifestations were varied without specific characteristics, and it may be confused with other lung diseases, especially pulmonary tumor. Occupying lesions and other types of lesions should be excluded prior to the diagnosis of OP. It is difficult to distinguish OP and lung cancer from clinical symptoms and imaging findings alone, 
and definite diagnosis typically requires pathological examination results.

Treatment with systemic corticosteroids is often effective; however, it typically requires a lengthy treatment period and is accompanied by adverse side effects. Available treatments at present have yet to achieve desirable therapeutic efficacy.

Considering the possibility that OP may coexist with lung carcinoma, small sample biopsy may not necessarily distinguish between the diseases, and large sample pathological examination may be required. Surgery is an adequate treatment, but may be excessive. At present, the thoracoscope is the most commonly used method.

Improvement of diagnostic techniques is required, and further research should be conducted to identify more effective therapeutic methods, in order to reduce or avoid trauma to patients from unnecessary surgeries.

\section{References}

1. Ding QL, Lv D, Wang BJ, Zhang QL, Yu YM, Sun SF, Chen ZB Ma HY and Deng ZC: Macrolide therapy in cryptogenic organizing pneumonia: A case report and literature review. Exp Ther Med 9: 829-834, 2015.

2. Narasimhaiah DH, Chakravorty I, Swamy R and Prakash D: Organising pneumonia presenting as acute life threatening pulmonary haemorrhage. BMJ Case Rep 2011: pii: bcr0320091689, 2011.

3. Zhao F, Yan SX, Wang GF, Wang J, Lu PX, Chen B, Yuan J, Zhang SZ and Wang YX: CT features of focal organizing pneumonia: An analysis of consecutive histopathologically confirmed 45 cases. Eur J Radiol 83: 73-78, 2014.

4. Epler GR, Colby TV, McLoud TC, Carrington CB and Gaensler EA: Bronchiolitis obliterans organizing pneumonia. N Engl J Med 312: 152-158, 1985.

5. Radzikowska E, Nowicka U, Wiatr E, Jakubowska L, Langfort R, Chabowski M and Roszkowski K: Organising pneumonia and lung cancer - case report and review of the literature. Pneumonol Alergol Pol 75: 394-397, 2007.

6. Melloni G, Cremona G, Bandiera A, Arrigoni G, Rizzo N, Varagona R, Muriana G, Carretta A, Ciriaco P, Canneto B and Zannini P: Localized organizing pneumonia: Report of 21 cases. Ann Thorac Surg 83: 1946-1951, 2007.

7. Xie S, Shen C, Zhang Y, Lu K, Hu F, Tan M, Lin H, Xu L, Yuan Q, Song $\mathrm{X}$ and Wang C: Cryptogenic organizing pneumonia associated with invasive pulmonary aspergillosis: A case report and review of the literature. Int J Clin Exp Pathol 7: 8637-8646, 2014.

8. Sanito NJ, Morley TF and Condoluci DV: Bronchiolitis obliterans organizing pneumonia in an AIDS patient. Eur Respir J 8: 1021-1024, 1995

9. Haro M, Vizcaya M, Texidó A, Aguilar X and Arevalo M: Idiopathic bronchiolitis obliterans organizing pneumonia with multiple cavitary lung nodules. Eur Respir J 8: 1975-1977, 1995.

10. Pérez de Llano LA, Soilán JL, García Pais MJ, Mata I, Moreda M and Laserna B: Idiopathic bronchiolitis obliterans with organizing pneumonia presenting with adult respiratory distress syndrome. Respir Med 92: 884-886, 1998.

11. Stey C, Truninger K, Marti D, Vogt P and Medici TC: Bronchiolitis obliterans organizing pneumonia associated with polymyalgia rheumatica. Eur Respir J 13: 926-929, 1999.

12. Alcolea S, Santiago Recuerda A and Prados MC: Cryptogenic organizing pneumonia and mediastinal lymphadenopathy. Arch Bronconeumol 40: 384, 2004 (In Spanish).

13. Arrabal Sánchez R, Mongil Poce R, Benítez Doménech A, Fernández de Rota Avecilla A and Fernández Bermúdez JL: Bronchiolitis obliterans organizing pneumonia and bronchogenic carcinoma coexisting in different parts of the lungs. Arch Bronconeumol 40: 141-143, 2004 (In Spanish).
14. Husain SJ, Irfan M, Zubairi AS and Salahuddin N: Rapidly-progressive bronchiolitis obliterans organising pneumonia. Singapore Med J 45: 283-285, 2004.

15. Hooi LN: Bronchiolitis obliterans organising pneumonia: A treatable condition. Med J Malaysia 60: 222-225, 2005.

16. Burke L and Jacob BK: Reversed halo sign in cryptogenic organising pneumonia. BMJ Case Rep 2010: pii: bcr0320102824, 2010.

17. Maimon N: A 47-year-old female with shortness of breath and 'reversed halo sign'. Eur Respir Rev 19: 83-85, 2010.

18. Eguchi T, Takasuna K, Fujiwara M and Yoshida K: Coexistence of a pulmonary adenocarcinoma with a focal organizing pneumonia. Interact Cardiovasc Thorac Surg 13: 444-446, 2011.

19. Lee J, Cha SI, Park TI, Park JY, Jung TH and Kim CH: Adjunctive effects of cyclosporine and macrolide in rapidly progressive cryptogenic organizing pneumonia with no prompt response to steroid. Intern Med 50: 475-479, 2011.

20. Pardo J, Panizo A, Sola I, Queipo F, Martinez-Peñuela A and Carias R: Prognostic value of clinical, morphologic, and immunohistochemical factors in patients with bronchiolitis obliterans-organizing pneumonia. Hum Pathol 44: 718-724, 2013.

21. Sheikh IA, Saadia N, Sheikh N and Culpepper-Morgan JA: Simultaneous diagnosis of cryptogenic organizing pneumonia and HIV in a 45 year old man. Am J Case Rep 13: 166-168, 2012.

22. Terada T: Autopsy findings of fatal cryptogenic organizing pneumonia. Int J Clin Exp Pathol 6: 1128-1131, 2013.

23. Utrilla Contreras C, Fernández-Velilla Peña M, García Río F and Torres Sánchez MI: Radiographic patterns in the diagnostic approach to organizing pneumonia. Rev Clin Esp (Barc) 214: 258-265, 2014 (In English, Spanish).

24. Sugiura K, Muro Y and Akiyama M: Solitary organizing pneumonia mimicking lung adenocarcinoma in systemic sclerosis. Arthritis Rheumatol 66: 2648, 2014

25. Zannoni S, Lorenzi C, Paoli M, Orso F, Torrini M, Rafanelli M, Toffanello G, Giordano A, Gori Sacco FC, Marchionni N and Ungar A: P-263: Cryptogenic organizing pneumonia: A rare syndrome even in the elderly. Case report. Eur Geriatr Med 6 (Suppl 1): S102, 2015.

26. Nakahara Y, Oonishi Y, Takiguchi J, Morimoto A, Matsuoka K, Imanishi N, Higashino T, Mimura R, Kawamura $\mathrm{T}$ and Mochiduki Y: Nontuberculous mycobacterial lung disease accompanied by organizing pneumonia. Intern Med 54: 945-951, 2015.

27. Morichika D, Miyahara N, Hotta K, Okamoto Y, Minami D, Irie M, Tanimoto Y, Kanehiro A, Tanimoto M and Kiura K: Invasive mucinous adenocarcinoma mimicking organizing pneumonia associated with mycobacterium fortuitum infection. Intern Med 53: 2795-2799, 2014.

28. National Comprehensive Cancer Network: NCCN clinical practice guidelines in Oncology: Lung Cancer Screening (Version 2.2015) https://www.nccn.org/professionals/physician_gls/f_ guidelines.asp. Accessed March 17, 2015.

29. Feng AN, Cai HR, Zhou Q, Zhang YF and Meng FQ: Diagnostic problems related to acute fibrinous and organizing pneumonia: Misdiagnosis in 2 cases of lung consolidation and occupying lesions. Int J Clin Exp Pathol 7: 4493-4497, 2014

30. Travis WD, Costabel U, Hansell DM, King TE Jr, Lynch DA, Nicholson AG, Ryerson CJ, Ryu JH, Selman M, Wells AU, et al: An Official American Thoracic Society/European Respiratory Society Statement: Update of the international multidisciplinary classification of the idiopathic interstitial pneumonias. Am J Respir Crit Care Med 188: 733-748, 2013.

31. Baque-Juston M, Pellegrin A, Leroy S, Marquette $\mathrm{CH}$ and Padovani B: Organizing pneumonia: What is it? A conceptual approach and pictorial review. Diagn Interv Imaging 95: 771-777, 2014.

32. Zhao ZR, Li Z, Situ DR and Ng CS: Recent clinical innovations in thoracic surgery in Hong Kong. J Thorac Dis 8 (Suppl 8): S618-S626, 2016.

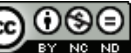

This work is licensed under a Creative Commons Attribution-NonCommercial-NoDerivatives 4.0 International (CC BY-NC-ND 4.0) License. 